\title{
Effect of Frequency on Energy Efficient Transceiver Design
}

\author{
Keshav Kumar, Bishwajeet Pandey, D M Akbar Hussain \\ Chitkara University Institute of Engineering \& Technology, Chitkara University, \\ Punjab, India \\ Department of Center of Energy Excellence Gyancity Research Lab Motihari, India \\ Department of Energy Technology, Aalborg University Esbjerg, Denmark \\ keshav.kumar@chitkara.edu.in,gyancity@gyancity.com,akh@et.aau.dk,
}

\begin{abstract}
We have observed the different chip power which are clustered on UART device, example IOs, leakage and total power. This through experiment is done at a frequency of $1 \mathrm{GHz}$, having a duty cycle of $50 \%$ and 1 ns time period. We did our experiment with Virtex 4, Virtex 5, Virtex 6, Spartan3 and Spartan 6 FPGA. In the experiment we found Spartan 6 uses the least amount of power among all the FPGA used in the experiment.
\end{abstract}

Keywords-FPGA, Power, Voltage, UART, Frequency.

\section{Introduction}

World is more relying on coal and natural resources for the generation and supply of electricity and this is possibly disturbing the global climate. Not only the world's population is rapidly increasing but the industrialization is also happening [1]. So to fulfil the everyone's need of energy is quiet difficult [2]. In order to minimize the energy consumption in communication network we have designed UART using different nanometers FPGA, which consumes least amount of energy. UART stands for Universal Asynchronous Receiver Transmitter. UART is running at a frequency of 1 GHZ, duty cycleof $50 \%$ and $1 \mathrm{~ns}$ time period. Duty cycle is the time period for which the signal is used. From the power and duty cycle relation $=(\mathrm{PW} / \mathrm{T}) * 100$, where $\mathrm{D}$ is duty cycle, PW is pulsewidth $\mathrm{T}$ is the time period of the signal. Data in UART is transmitted at a specific frequency known as Baudrate [3]. The time procedure involved in UART is shown in figure 1 the data is sampled when the baud rate of receiever and transmitter matches perfectly.

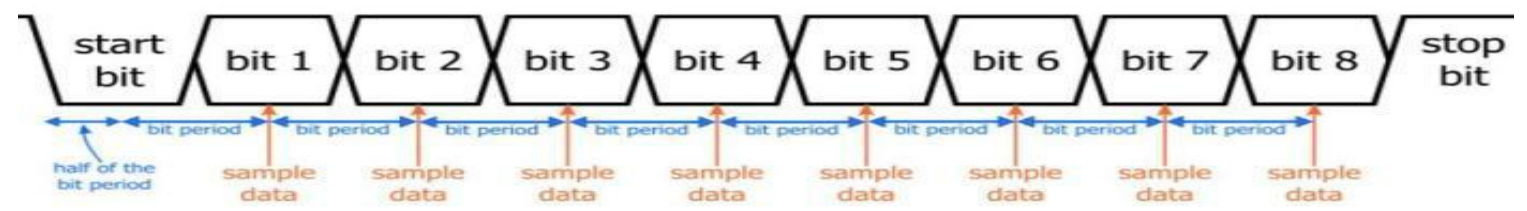

Figure 1. Data transfer in UART

\section{Related Work}

T.Taun ET. al. [4] a low power 90nm FPGA is designed by the authors, which is used for the application of battery powered devices. B. Pandey ET. al. [5] authors designed 
a system using Virtex-6 FPGA, which checks the reduction of leakage power with different IO standards. In this design dynamic voltage scaling is also done in Vedic multiplier. B. Pandey ET. al. [6] energy efficient Gurumukhi Unicode Reader (GUR) is designed by the authors on FPGAs, which is done at a frequency $0 \mathrm{f} 1 \mathrm{GHz}$ by varying IO standards. Md. H. Rais ET. al. [7] with the help of Virtex-5 FPGA authors have designed an efficient hardware for advanced encryption of cryptographic algorithm. K. Rrcan ET. al. [8] low energy high performance hardware is demonstrated by the researchers, which is used for high efficiency video encoding using Virtex-6 FPGA. But in our research we have designed an energy efficient transceiver using different nanometer technology FPGA which is operated at $1 \mathrm{GHz}$ frequency.

\section{Experimental Section}

The experiment we performed is done on XILINX 14.1 ISE design. The different FPGA used are Virtex-4, Virtex 5, Virtex-6, Spartan-3, and Spartan 6. The code of UART is written in Verilog Hardware Description Language. We have tested UART power on $\mathrm{X}$ power Analyzer tool in XILINX simulator at $1 \mathrm{GHZ}$ frequency, having $1 \mathrm{~ns}$ time period.

\section{Explanation}

The power table of Virtex-4, Virtex-5 and Virtex-6 FPGA is shown in table1.

Table 1. Supply power of Virtex 4, Virtex 5 and Virtex 6 FPGA

\begin{tabular}{|l|l|l|l|}
\hline Supply Power & Virtex 4 & Virtex 5 & Virtex 6 \\
\hline IOs & 0.011 & 0.014 & 0.017 \\
\hline Leakage & 0.167 & 0.321 & 0.027 \\
\hline Total & 0.204 & 0.350 & 0.063 \\
\hline
\end{tabular}

The power table of Spartan- 3 and Spartan- 6 is shown in table2.

Table 2. Supply power of Spartan 3 and Spartan 6 FPGA

\begin{tabular}{|c|c|c|}
\hline Supply Power & Spartan 3 & Spartan 6 \\
\hline IOs & 0.017 & 0.025 \\
\hline Leakage & 0.027 & 0.014 \\
\hline Total & 0.063 & 0.055 \\
\hline
\end{tabular}

\section{POWER ANALYSIS}

\section{A. Power Analysis of Virtex 4 v/s Virtex 5 v/s Virtex 6}

At $1 \mathrm{GHz}$ frequency Virtex 4 consumes least amount of IOs power. But for leakage power and total power Virtex 6 is power efficient. The power comparison shown in figure 2 . 


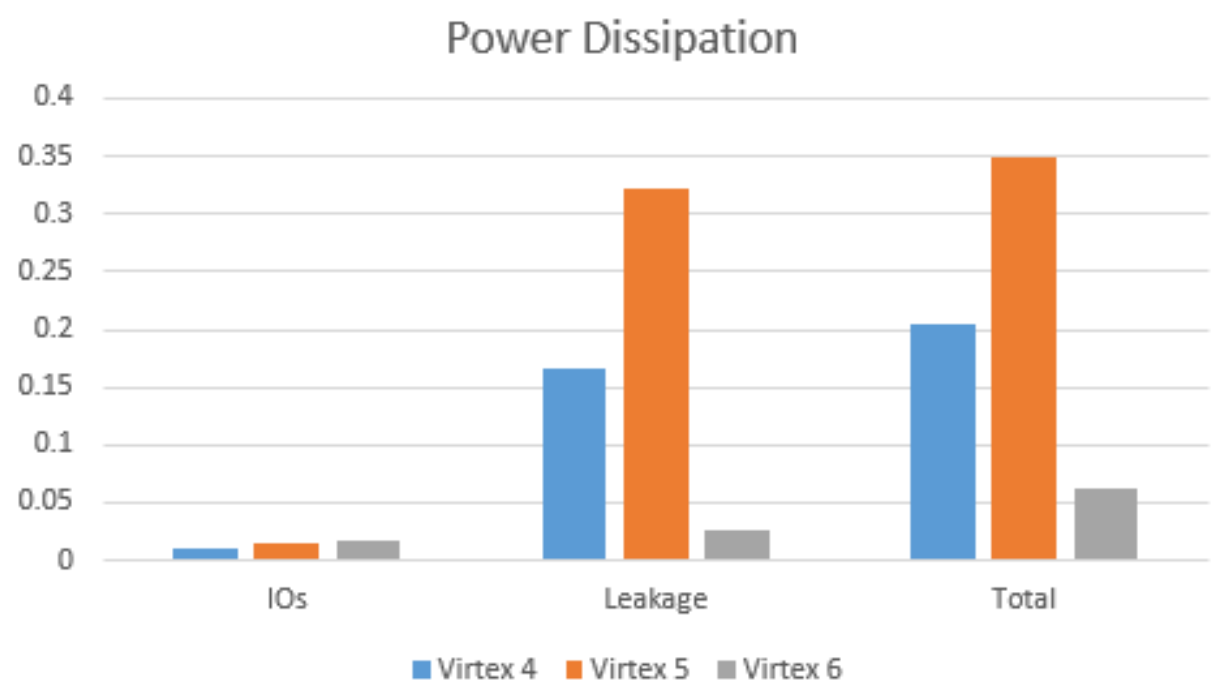

Figure 2. Power Analysis of Virtex-4 v/s Virtex-5 v/s Virtex-6

B. Power Analysis of Spartan 3 and Spartan 6

When frequency is $1 \mathrm{GHz}$ Spartan-6 consumes least amount of leakage and total power in comparison to Spartan-3 and for IOs power Spartan-3 requires least amount of power. The different power comparison is shown in Figure 3.

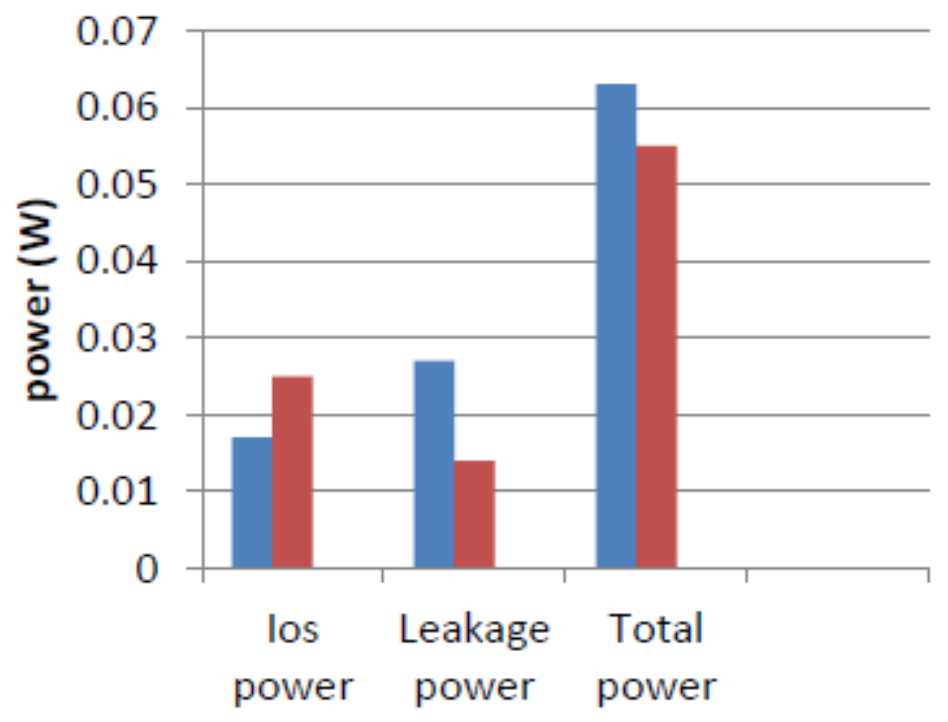

Figure 3. Power Analysis of Spartan-3 (Blue Color) and Spartan-6 (Red Color)

C. Power Analysis of Virtex 4 v/s Virtex 5 v/s Virtex 6 v/s Spartan 3 and Spartan 6.

When we compare all the FPGA at 1 GHZ frequency we found that Spartan 6 FPGA consumes the least amount of leakage power and total power but for IOs power Virtex 4 is most power efficient.S

\section{Results}


Gyancity Journal of Engineering and Technology,

Vol.5, No.2, pp. 14-18, July 2019

ISSN: 2456-0065 DOI: 10.21058/gjet.2019.52002 
It is observed that at $1 \mathrm{GHz}$ frequency Spartan 6 FPGA is most power efficient for leakage and total power. The percentage variation in leakage power and total power with Virtex 4, Virtex5, Virtex 6 and Spartan 3 is as 91.61\%, 95.638\%, 48.148\%, 48.148 and $73.039 \%, 84.285 \%, 12.698 \%, 12.698 \%$ respectively. For IOs power Virtex 4 is power efficient, Virtex 4 consumes $21.42 \%$ less power than Virtex 5, 54.54\% less power than Virtex 6, 54.54\% less power than Spartan 3 and $56 \%$ less power than Spartan 6.

\section{Conclusion}

We are comparing the power utilization of Spartan-3 and Spartan-6 FPGA by increasing the voltage of UART, we found that in case of IOs power Spartan-3 FPGA is more power efficient than Spartan-6 FPGA. And for leakage and total power Spartan6 FPGA should be used because it consumes less power than Spartan-3 FPGA. The power analysis of UART is done on Xilinx 14.1 ISE Design software and code of UART is written in Verilog module. After analyzing the results we can say that Spartan6 FPGA is more reliable over Spartan-3 FPGA.

\section{References}

[1] www.technologyreview.com/s/54209/indias-energy-crisis

[2] www.economictimes.indiatimes.com/indias-loomimg-powercrisis/articleshow/51051903.com

[3] www.allaboutcircuits.com/technical-articles/the-uart-baud-rate-how-accurate-does-it-need-tobel

[4] T. Taun, S. Kao, A. Rahman, S. Das, and S. Trimberger. "A 90nm low-power FPGA for batterypowered applications." In Proceedings of the 2006 ACM/SIGDA 14th international symposium on Field programmable gate arrays, pp. 3-11. ACM, 2006.

[5] B. Pandey, Md A. Rahman, A. Saxena, and B. Das. "Leakage power reduction with various IO standards and dynamic voltage scaling in vedic multiplier on Virtex-6 FPGA." Indian Journal of Science and Technology 9, no. 25 (2016).

[6] B. Pandey, and G. Singh. "Simulation of cmos io standard based energy efficient gurmukhi Unicode Reader on FPGA." In Computational Intelligence and Communication Networks (CICN), 2014 International Conference on, pp. 917-920. IEEE, 2014.

[7] Md H. Rais, and S. M. Qasim. "Efficient hardware realization of advanced encryption standard algorithm using Virtex-5 FPGA." International Journal of Computer Science and Network Security 9, no. 9 (2009): 59-63.

[8] K. Ercan, Y. Adibelli, and I. Hamzaoglu. "A high performance and low energy intra prediction hardware for high efficiency video coding." In Field Programmable Logic and Applications (FPL), 2012 22nd International Conference on, pp. 719-722. IEEE, 2012). 